\begin{tabular}{llll}
\hline Submission: 26/Jan/2021; & $1^{\text {st }}$ round notif.: 05/May/2021; & New version: 25/May/2021; & $2^{\text {nd }}$ round notif.: 29/Jun/2021; \\
Camera ready: 15/Jul/2021; & Edition review: 20/Jul/2021; & Available online: 06/Aug/2021; & Published: 06/Aug/2021;
\end{tabular}

\title{
Avaliação da interação em MOOC: uma análise integrada sobre as causas de abandono dos estudantes
}

\author{
Title: Assessment of interaction in MOOC: an integrated analysis of the causes of student \\ dropout
}

\author{
Beatriz B. do Rêgo \\ Programa de Pós- \\ graduação em Ciência da \\ Computação (PGCOMP) \\ Universidade Federal da \\ Bahia (UFBA) \\ beatrizbr@ufba.br
}

\author{
Filipe A. Garrido \\ Programa de Pós- \\ graduação em Ciência da \\ Computação (PGCOMP) \\ Universidade Federal da \\ Bahia (UFBA) \\ filipe.garrido@ufba.br
}

\author{
Jean C. S. Rosa \\ Programa de Pós- \\ graduação em Ciência da \\ Computação (PGCOMP) \\ Universidade Federal da \\ Bahia (UFBA) \\ jean.rosa@ufba.br
}

\author{
Ecivaldo de Souza Matos \\ Instituto de Computação \\ Universidade Federal da \\ Bahia (UFBA)
}

ecivaldo@ufba.br

\section{Resumo}

Os Massive Open Online Courses (MOOC) surgiram como uma abordagem educacional inovadora, por meio da prestação de cursos online sem número predefinido de estudantes. Porém, cursos MOOC têm sofrido com alta taxa de abandono dos seus estudantes. Entre as diversas causas de abandono indicadas na literatura estão o idioma da plataforma e a insuficiência de habilidade do usuário para o uso de plataformas MOOC. Este artigo apresenta a correlação dos resultados da avaliação da qualidade da interação de uma plataforma MOOC com as causas gerais desse abandono identificadas na literatura. A avaliação foi realizada a partir dos resultados dos métodos de Percurso Cognitivo (PC) e do Método de Inspeção Semiótica (MIS). Os resultados apontam que a qualidade da interação pode influenciar (direta ou indiretamente) a ocorrência de algumas causas gerais de abandono dos estudantes. É recomendada a execução de outros métodos de avaliação para análise mais profunda sob a perspectiva do estudante.

Palavras-Chave: Massive Open Online Courses; abandono; permanência; Percurso Cognitivo; Método de Inspeção Semiótica.

\begin{abstract}
Massive Open Online Courses (MOOC) emerged as an innovative educational approach by providing online courses without a predefined number of students. However, MOOC courses have suffered from a high dropout rate from their students. Among the various causes of abandonment indicated in the literature are the platform's language and the insufficiency of the user's ability to use MOOC platforms. This article presents the correlation of the evaluation results of the quality of the interaction of a MOOC platform with the general causes of abandonment identified in the literature. The evaluation was performed based on the Cognitive Pathway (CP) results and the Semiotic Inspection Method (SIM) methods. The results show that the interaction quality can influence (directly or indirectly) the occurrence of some general causes of student dropout. It is recommended to use other methods of assessment for further analysis from the student's perspective.
\end{abstract}

Keywords: Massive Open Online Courses; dropping out; retention; Cognitive Walkthrough; Semiotic Inspection Method.

Cite as: Do Rêgo, B. B., Garrido, F. A., Rosa, J. C. S., \& Matos, E. S. (2021). Avaliação da interação em MOOC: uma análise integrada sobre as causas de abandono dos estudantes. Revista Brasileira de Informática na Educação, 29, 846-862. DOI: 10.5753/RBIE.2021.29.0.846 


\section{Introdução}

Os Massive Open Online Courses (MOOC) são um tipo específico de curso online, aberto (que não necessita cadastro) e massivo (sem dimensão pré-definida de estudantes). Um de seus principais objetivos é a democratização da educação por meio do compartilhamento das experiências individuais de cada estudante (He et al., 2015; Baloi, 2009). Entretanto, os cursos MOOC têm apresentado alta taxa de abandono pelos estudantes (He et al., 2015; Clow, 2013; Alario-Hoyos et al., 2013; Baloi, 2009; Onah, 2014).

Embora milhares de estudantes se inscrevam em cursos MOOC, a taxa de conclusão, em média, para a maioria dos cursos é de 13\% (Onah, 2014). Algumas causas gerais para o abandono dos estudantes encontradas na literatura estão relacionadas à (i) escassez de tempo por parte dos participantes, (ii) insuficiência de habilidade para uso das tecnologias, (iii) dificuldade em expor ideias de modo clara em comunicação escrita, (iv) privação da interação entre aluno e professor e o (v) dificuldades no entendimento do idioma da plataforma de suporte ao curso (Gomez-Zermeno \& De La Garza, 2016; Bittencourt, 2011; Dinniz, 2007).

Para o usuário usufruir melhor do suporte computacional ofertado, é desejável que os recursos tecnológicos sejam de fácil uso (usabilidade) e metacomuniquem as suas concepções e intenções de interação (comunicabilidade) (Barbosa \& Silva, 2010, p. 36). Neste artigo são apresentados os resultados da avaliação de interação de um curso MOOC, de modo a correlacionar os resultados com as causas gerais de abandono de estudantes de cursos MOOC encontradas na literatura.

Para avaliar a interação, foram selecionados dois métodos de inspeção: o método de Percurso Cognitivo (PC) e o Método de Inspeção Semiótica (MIS). O método de PC foi selecionado por ser um método de inspeção fundamentado na Engenharia Cognitiva, cujo objetivo foi avaliar a usabilidade e a capacidade de aprendizado do usuário no decorrer da interação. Por sua vez, o MIS é um método da Engenharia Semiótica (De Souza \& Leitão, 2009) com o qual avalia-se a comunicabilidade de sistemas computacionais interativos por meio de inspeção.

Sob perspectivas distintas de qualidade em seu objetivo de avaliação, o uso de dois métodos com perfis de avaliação distintos permitiu a assunção de uma estratégia holística para avaliar uma plataforma MOOC e direcionar esforços para sua melhoria/adequação em mais de um aspecto de qualidade da Interação Humano-Computador (IHC).

Este artigo está organizado em seis seções. Na segunda seção são apresentados os conceitos fundamentais sobre MOOC e alguns dados sobre o abandono de estudantes nessa modalidade. A seção seguinte apresenta a metodologia do estudo. Na quarta seção são apresentados os resultados das aplicações do MIS e do PC. Na seção cinco tem-se a discussão dos resultados, em que eles são correlacionados com as causas gerais de abandono em cursos MOOC. Por fim, a última seção apresenta as considerações finais e sugestões de trabalhos futuros.

\section{Causas de Abandono de MOOC}

O termo MOOC é um acrônimo para Massive Open Online Courses, abordagem desenvolvida por Cormier e Alexander em 2008 para descrever um tipo peculiar de cursos online (Grainger, 2013). O primeiro curso foi desenvolvido por Stephen Downes e George Siemens com princípios conectivistas. O conectivismo é uma teoria de aprendizagem que possui como premissa básica a difusão de conhecimento por meio de uma rede de conexões, na qual a aprendizagem consiste na capacidade de construir e percorrer essas redes (Downes, 2012).

Há na literatura, dois principais modos de classificação de MOOC, ambos baseados em diferentes ênfases pedagógicas e modelos organizacionais (Grainger, 2013): 
- $\quad c M O O C$ : apresenta abordagem conectivista, baseando-se nas conexões entre os membros e no compartilhamento de conteúdo entre eles, semelhante à proposta de educação aberta.

- $\quad x M O O C$ : fortemente apoiado por materiais multimídia, principalmente vídeos, além disso utiliza quiz e fórum para avaliar os participantes dos cursos.

No decorrer dos anos notou-se evolução progressiva dos MOOC, em 2011 constatou-se a expansão do uso de MOOC em todo o mundo e foram introduzidos na consciência pública (Baturay, 2015). Consequentemente, a partir dessa evolução, 2012 ficou conhecido como o ano dos MOOC (Pappano, 2012). Algumas iniciativas foram elaboradas com o intuito de apoiar essa nova modalidade de aprendizagem online (Grainger, 2013); uma delas foi a criação de plataformas que proporcionem suporte aos MOOC. Dentre as mais populares, destacam-se Coursera ${ }^{1}$, Udacity $^{2}$ e EDx ${ }^{3}$.

Uma das principais críticas aos MOOC está relacionada à baixa taxa de permanência dos estudantes (Grainger, 2013). Alguns dados mostram que existe alto índice de abandono em cursos MOOC. O primeiro curso MOOC disponibilizado pela plataforma MITx ${ }^{4}$ chegou a possuir 150 mil estudantes matriculados; porém, apenas 7\% concluíram os cursos (Clow, 2013). O primeiro curso MOOC oferecido pela plataforma Coursera contabilizou 51.306 estudantes inscritos, mas somente 795 concluíram os cursos, correspondendo a 1,5\% dos estudantes originalmente inscritos (He et al., 2015).

Belanger et al. (2013) relatam a experiência do planejamento, desenvolvimento e entrega de um MOOC desenvolvido em parceria da Universidade de Duke com a plataforma Coursera. Nesse estudo identificou-se três obstáculos para que os estudantes conseguissem concluir o curso MOOC. Um deles foi o pouco tempo disponível para realizar o curso, citada pelos estudantes como um fator significativo de impedimento para completar o curso. Os outros dois fatores são formação insuficiente em matemática ou intenção de apenas ver as aulas desde o início (Belanger et al., 2013).

Nawrot e Doucet (2014) também identificaram algumas causas de abandono em MOOC. O principal motivo para o abandono de estudantes em MOOC identificado pelos autores foi o mau gerenciamento do tempo. Esse dado foi confirmado por $68,9 \%$ dos participantes que responderam à pesquisa. Outros autores reafirmam que a falta de tempo por parte dos estudantes é fator determinante para o abandono de cursos MOOC (Zheng et al., 2015; Fini, 2009; Burge, 2015).

Para além da gestão do tempo, Do Rêgo et al. (2017b) ao avaliarem uma plataforma de suporte a cursos MOOC, perceberam que rupturas de comunicabilidade da interface interferiam no modo como o estudante usava a plataforma. As autoras identificaram que essas rupturas inibem os estudantes, que afirmaram deixar de utilizar determinadas funcionalidades da plataforma por não saber como usar ou não as entender. Em ambientes de MOOC, caso um(a) estudante apresente as dificuldades de interação que promovam rupturas de comunicabilidade, ele(a) pode vir a abandonar o curso (Do Rêgo et al., 2017a). Ainda que cursos MOOC sejam considerados "abertos", os estudantes precisam de uma competência fundamental: habilidades no uso de tecnologias da informação (Fini, 2009).

Outro aspecto confirmado no estudo de Do Rêgo et al. (2017a) foram as falhas de tradução da interface. $\mathrm{O}$ usuário configura o idioma desejado, mas em alguns casos apenas algumas porções da plataforma são traduzidos para o idioma selecionado pelo usuário. O restante da plataforma

\footnotetext{
1 https://pt.coursera.org/

2 https://br.udacity.com/

3 https://www.edx.org/

4 https://www.edx.org/school/mitx
} 
permanece no idioma de origem de desenvolvimento, geralmente o inglês. Caso o usuário não possua familiaridade com o idioma, poderá abandonar o curso.

Gomes-Zermero e De La Garza (2016) destacam que um dos motivos de desânimo está associado à ausência de tutoriais para orientação dos estudantes. É essencial que os usuários entendam as mensagens da plataforma, para que eles possam fazer melhor uso e tirar melhor proveito de todas as funcionalidades do sistema (Silveira et al., 2003). Por isso, a ausência de tutorial para orientar os estudantes está diretamente ligada à qualidade da metacomunicação e possivelmente ao abandono dos estudantes.

Orientando-se pelas causas gerais de abandono de MOOC indicadas na literatura acadêmico-científica, procedimentos metodológicos para condução de uma avaliação holística da interface de uma plataforma de MOOC foram concebidos.

\section{Metodologia}

Este estudo foi realizado em três etapas: (i) execução do método Percurso Cognitivo (PC) para avaliar a usabilidade da interface de um curso disponível na plataforma Coursera; (ii) execução do Método de Inspeção Semiótica (MIS) da interface de um curso disponível na plataforma Coursera; e (iii) correlação dos resultados com os dados apresentados na execução do MIS e do PC com as causas de abandono de estudantes da plataforma Coursera.

Para realizar este estudo foi selecionado por conveniência um dos cursos da plataforma Coursera, intitulado Successful Negotiation: Essential Strategies and Skills (Negociações de Sucesso: estratégias e habilidades essenciais). A plataforma Coursera foi escolhida por apresentar, segundo a literatura, os maiores índices de abandono em seus cursos (He et al., 2015; Clow, 2013; Alario-Hoyos et al., 2013; Almeida et al., 2013; Bartolomé, 2015).

Diante da impossibilidade de avaliar mais plataformas, tanto pelo tempo quanto pelo custo, optamos por uma plataforma que apresentasse número expressivo de alunos e índices elevados de abandono, para ampliar o impacto das possíveis melhorias indicadas como resultado do estudo. Além disso, as principais plataformas de MOOC apresentam diversas similaridades (Do Rêgo et al., 2019), isso pode contribuir para a generalização de algumas das soluções apontadas no estudo para além do Coursera. Por fim, os aspectos do sistema que foram avaliados tratam essencialmente de recursos disponíveis na plataforma, independente do curso escolhido.

As execuções do PC e MIS foram realizadas por três especialistas em Interação HumanoComputador (IHC) sob orientação de um pesquisador experiente na área, todos autores do artigo. Os autores são graduados em Engenharia de Software, Análise de Sistemas e Sistemas para Internet e Ciência da Computação; atuam como pesquisadores na área de Design de Interação, Design Participativo e Engenharia Semiótica; realizaram avaliações de usabilidade e comunicabilidade em projetos anteriores.

As inspeções foram realizadas em conjunto e uma discussão coletiva ocorreu para unificar os resultados apresentados por similaridades. A intenção foi harmonizar o discurso resultante da avaliação feita pelos dois métodos em uma percepção única dos problemas encontrados e que as soluções fossem válidas para ajustes e melhorias do sistema sob a perspectiva da comunicabilidade e usabilidade, sem distinção entre ambas.

\subsection{Método de Inspeção Semiótica}

O Método de Inspeção Semiótica (MIS) tem o objetivo de construir "uma versão integrada da mensagem de metacomunicação, identificando inconsistências e ambiguidades" (Salgado \& De Souza, 2007), avaliando a emissão da metamensagem; entendendo a metacomunicação como o 
processo de comunicação entre o designer e usuário por meio da interface do sistema projetado pelo designer ${ }^{5}$.

Os cenários criados para a avaliação são (Do Rêgo et al., 2017a):

1. Cadastro do usuário: a realização desta tarefa possibilitará ao estudante ter acesso a todos os cursos da plataforma Coursera.

2. Seleção do curso: como o objetivo deste estudo é avaliar a interação da plataforma MOOC, o estudante ao realizar esta tarefa terá que selecionar um curso que seja do tipo MOOC, pois a plataforma Coursera também disponibiliza cursos online que não são MOOC. Então o curso selecionado foi o intitulado Successful Negotiation: Essential Strategies and Skills (Negociações de sucesso: estratégias e habilidades essenciais)

3. Início do curso: ao realizar esta tarefa, o estudante iniciará suas aulas e poderá realizar as atividades propostas.

4. Participação no fórum: nesta tarefa o estudante contribuirá com outros participantes do curso, compartilhando informações e experiências.

Esse método investiga três tipos de signos codificados na interface, os signos estáticos, dinâmicos e metalinguísticos. O pesquisador/avaliador deve interpretar ao longo da inspeção os signos identificados, com o objetivo de reconstruir a metamensagem do designer utilizando o modelo padrão. O último passo é comparar os resultados das três metamensagens construídas, para que o avaliador possa buscar intencionalmente por significados contraditórios, inconsistentes ou ambíguos.

Segundo Barbosa e Silva (2010), os signos estáticos expressam o estado do sistema, cujo significado é interpretado independentemente de relações causais e temporais da interface, como por exemplo: os itens de menu, os botões de uma barra de ferramenta, os campos e os botões de um formulário, entre outros. Os signos dinâmicos são signos que expressam o comportamento do sistema, envolvendo aspectos temporais e causais da interface; estão vinculados à própria interação e devem ser interpretados fazendo referência a ela. Por fim, os signos metalinguísticos são aqueles usados pelo designer para comunicar explicitamente aos usuários os significados que ele atribuiu para os demais signos codificados na interface e como eles devem ser usados.

Ao conceber a metacomunicação, o designer se baseia na metamensagem parafraseada nos termos descritos a seguir.

Este é o meu entendimento, como designer, de quem você, usuário, é, do que aprendi que você quer ou precisa fazer, de que maneiras prefere fazer, e por quê. Este, portanto, é o sistema que projetei para você, e esta é a forma como você pode ou deve utilizá-lo para alcançar uma gama de objetivos que se encaixam nesta visão.

O presente estudo utiliza os dados da aplicação do MIS apresentados no estudo de Do Rêgo et al. (2017a) para que não fosse necessária uma nova aplicação do método, uma vez que o ambiente é exatamente o mesmo. A aplicação do percurso cognitivo expande a inspeção sob o prisma da usabilidade para que os ajustes do ambiente de MOOC, em nosso caso Coursera, seja o mais abrangente possível em relação aos critérios de qualidade indicados pela IHC.

\subsection{Percurso Cognitivo}

O Percurso Cognitivo (PC) é um método de inspeção fundamentado na Engenharia Cognitiva, cujo objetivo é avaliar a usabilidade e a capacidade de aprendizado do usuário no decorrer da interação (humano-computador). Como método de inspeção, apoia-se na avaliação de quanto a

\footnotetext{
${ }^{5}$ A Engenharia Semiótica entende o conceito de designer como o interlocutor do diálogo.
} 
interface facilita a exploração e o aprendizado sobre o software por parte do usuário (Salgado et al., 2006).

Os passos necessários para a realização do PC são divididos em: (i) percorrer a interface de acordo com a sequência de ações necessárias para realizar as tarefas definidas pelos avaliadores; e posteriormente, (ii) para cada tarefa criada, analisar se o usuário executaria a ação corretamente, respondendo e justificando a resposta às seguintes perguntas (Barbosa \& Silva, 2010, p. 324):

1. O usuário vai tentar atingir o efeito correto? (Vai formular a intenção correta?)

2. O usuário vai notar que a ação correta está disponível?

3. O usuário vai associar a ação correta com o efeito que está tentando atingir?

4. Se a ação for executada corretamente, o usuário vai perceber que está progredindo na direção de concluir a tarefa?

Ao executar esta etapa, espera-se que os resultados sejam sintetizados sobre o que o usuário precisa saber a priori para realizar as tarefas, o que o usuário deve aprender enquanto realiza as tarefas e sugestões de correções para os problemas encontrados. A partir dessa sintetização será gerado um relatório consolidado com os problemas encontrados e sugestões de correção (Barbosa \& Silva, 2010).

\subsubsection{Preparação do Percurso Cognitivo}

Antes de executar o PC é necessário criar uma lista de tarefas e identificar o perfil dos usuários. A lista de tarefas guiará a inspeção dos avaliadores, assim, as tarefas utilizadas foram as mesmas criadas no estudo que retornou rupturas encontradas pelo MIS (Do Rêgo et al., 2017a). As tarefas dividiram-se em: (i) cadastro do usuário, (ii) seleção de curso, (iii) início do curso e (iv) participação no fórum.

O perfil definido para os usuários foi o mesmo perfil semiótico elaborado por intermédio do MIS com a reconstrução da metamensagem. Apresentando o usuário como:

um estudante que procura cursos online disponiveis na internet. Porém, não qualquer curso online, um estudante que procura um curso do tipo MOOC. E que está acostumado a interagir com outros estudantes por meio dos fóruns de discussões. Um usuário que utiliza de conhecimentos adquiridos em outras interfaces de sistemas para "navegar" na interface do curso MOOC (Do Rêgo et al., 2017a).

\section{Resultados}

Os resultados estão divididos entre duas subseções, a subseção com os resultados do Método de Inspeção Semiótica e a subseção com os resultados do Percurso Cognitivo.

\subsection{Resultados do Método de Inspeção Semiótica}

Para melhor compreensão, as metamensagens são apresentadas fragmentadas, sendo as primeiras descritas referentes aos signos metalinguísticos (Do Rêgo et al., 2017a).

[Você é um usuário que possui um objetivo e está à procura dos melhores cursos onlines disponíveis na internet para poder alcançá-lo]. A página inicial do Coursera, ao ser aberta, chama a atenção do leitor à maior frase escrita na interface da página, "Take the world's best course, online", na qual a plataforma informa que possui os melhores cursos do mundo (ver Figura 1). 
Isso mostra que o designer deseja informar para o usuário que sabe que ele está buscando cursos online e ele encontrou o lugar onde estão os melhores cursos.

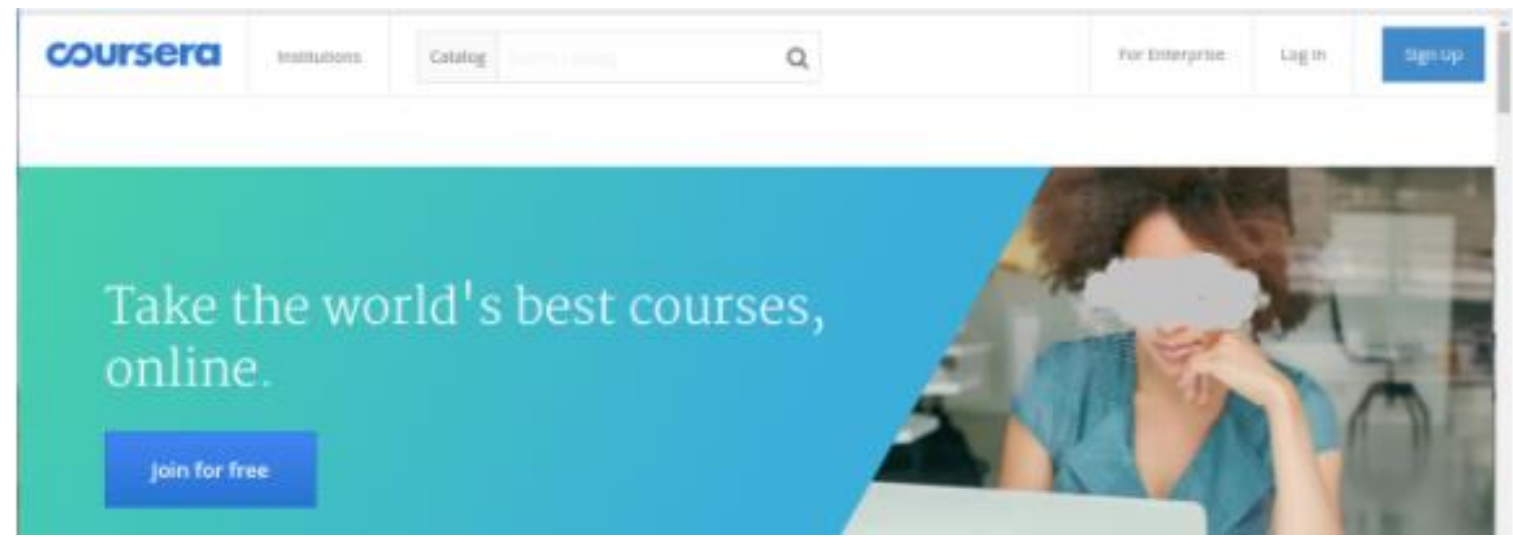

Figura 1: Página inicial do Coursera.

[É uma pessoa que conhece o termo MOOC e está procurando um curso que é classificado como MOOC. Mas entende que a plataforma que escolheu para buscar o curso não possui uma categoria especifica para MOOC]. As tarefas foram criadas com o objetivo de avaliar um MOOC, mas na plataforma Coursera não existe uma categoria específica para MOOC, dentre as categorias de curso existentes. O que acaba por dificultar a busca de cursos MOOC pelos usuários.

[Além disso, você é uma pessoa acostumada a inspecionar um site antes de começar a executar a tarefa que o levou ao mesmo, prestando atenção nos guias da central de ajuda]. $\mathrm{O}$ designer assume que o usuário está acostumado a verificar o site antes de iniciar o cadastro da plataforma. Pois, na página inicial, em sua parte inferior, encontra-se um pequeno tutorial de como funciona a execução dos cursos disponíveis.

[Uma pessoa que sempre buscará a central de ajuda quando necessário. Você entende que o idioma da plataforma é o inglês e percebe ao efetuar logon no sistema que estava no caminho certo para encontrar o que buscava]. O designer disponibiliza vários atalhos para a central de ajuda em partes diferentes do site, para que essa opção esteja sempre ao alcance do usuário. É possível perceber que o designer assume que o usuário é conhecedor de outro idioma, pelo fato de mesmo com a mudança de configuração o site oscilar entre dois idiomas - o inglês (padrão) e o português, configurado pelo usuário. Quando o usuário confirma o cadastro, ele é direcionado a uma página, na qual o designer reafirma que o usuário está no caminho certo para alcançar seu objetivo (Figura 2), ao dizer "Não importa o que você queira fazer, temos os cursos para você chegar lá” (tradução nossa).

\section{Welcome to Coursera}

Figura 2: Imagem de motivação da plataforma Coursera.

[Além disso, você é uma pessoa organizada que se planeja ao realizar as tarefas que se propõe até alcançar seu objetivo, mas precisa sempre de lembretes e informações sobre o decorrer do curso para que possa conclui-lo, como também incentivos para participar ativamente dos fóruns de discussões sobre cada módulo concluído durante o MOOC]. Ao curso ser selecionado pelo usuário, o designer disponibiliza o cronograma referente ao conteúdo, contendo a quantidade de vídeos e leituras que o usuário deverá realizar para concluir o módulo. Nesse sentido, a preocupação do designer em que o usuário conclua o curso pode ser vista nas frases encontradas na interface, como: "Para concluir o curso você (usuário), deve ser aprovado em todas 
as tarefas" ou "O curso avança rapidamente, por isso é importante manter-se em dia". Caso o usuário não cumpra esses dois "pedidos" poderá perder o cronograma do curso MOOC (ver Figura 2).

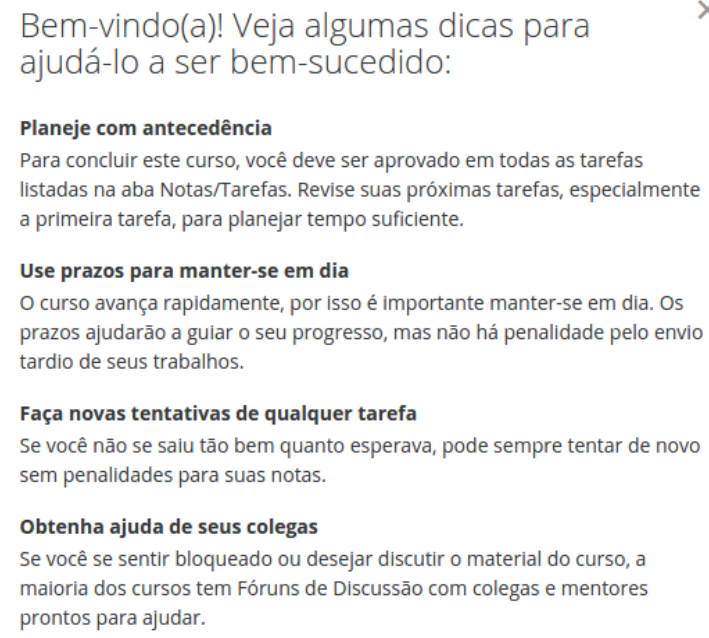

Figura 3: Informes início do curso.

Abaixo apresenta-se a metamensagem referente à inspeção dos signos estáticos, seguindo o mesmo formato fragmentado com as evidências da inspeção:

[Você é um usuário que utiliza conhecimentos adquiridos de outras interfaces. Sabendo disso, utilizamos símbolos e cores comuns do seu cotidiano para te guiar]. O designer utiliza padrões de elementos de interface para situar o usuário, como a forma retangular e as cores azul e branco para definir os botões. Ao realizar o cadastro, o usuário encontra desenhos associados ao objetivo que o levou a procurar um curso. Como, por exemplo, o desenho de um foguete que o designer faz associação com subida e elevação, numa referência à progressão na carreira profissional. Como também o desenho de um coração sobre um livro, em que o usuário está buscando cursos pelo amor (prazer) de estudar.

[Você é um usuário que deseja realizar um curso MOOC, mas não quer ser cobrado por isso]. Na página inicial do Coursera o designer disponibiliza para o usuário dois botões, para que ele possa se cadastrar. Em um desses botões, o designer informa para o usuário, potencial estudante, que não será cobrado nenhuma taxa para que ele participe do curso. Quando o usuário seleciona o curso, o designer automaticamente sugere outros cursos que possam despertar o interesse do usuário. Nesse caso o designer utiliza imagens para ajudar o usuário a distinguir o contexto dos cursos sugeridos. Por exemplo, o curso de Interaction Design, que tem como imagem um computador com vários "signos" saindo da tela, como se estivessem conversando com ele. Quando o usuário inicia o curso MOOC, encontra o símbolo de uma bandeira que o designer utilizou para que o usuário solicite ajuda, um sinalizador para quando algo não estiver ocorrendo como deveria. Na realização dessa tarefa foi notado que a legenda não estava disponível e os avaliadores recorreram a este recurso para solucionar o problema (ver Figura 4).

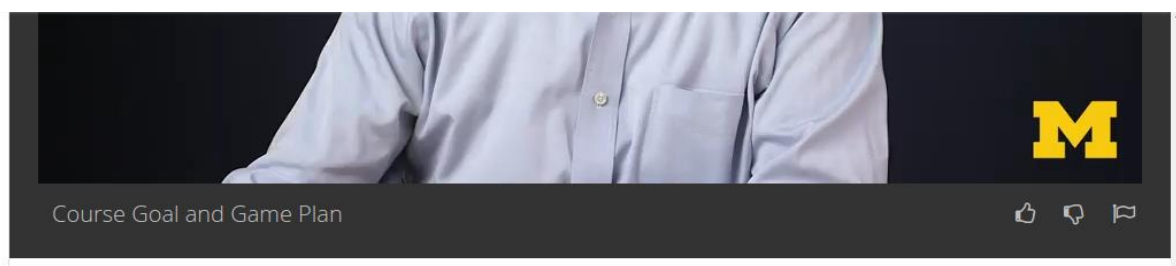

Figura 4: Signos do vídeo da aula. 
E por último, a seguir, temos a metamensagem reconstruída a partir dos signos dinâmicos para as tarefas realizadas:

[Você é um usuário que antes de iniciar um curso gostaria de ter uma prévia de como esse curso iria ser executado]. Como já informado nos signos metalinguísticos, o designer disponibiliza um pequeno tutorial dos passos para a execução de um curso. Ao usuário passar o cursor do mouse sobre uma das três opções, o texto é alterado dinamicamente para instigar o usuário a selecionar essa opção.

[É um usuário que ao ter dúvida sobre os cursos de uma determinada área, busca instantaneamente a descrição sem precisar mudar de página]. Antes de iniciar o curso, o usuário tem a opção de informar qual foi o seu objetivo ao se cadastrar no Coursera e selecionar áreas de interesse que estão listadas. Caso o usuário não reconheça o símbolo que está associado à área, ele pode posicionar o cursor do mouse sobre esse símbolo para que, dinamicamente, a figura revele uma breve descrição sobre a área em questão.

[Você precisa receber sinais de confirmação ao configurar o idioma da plataforma, para que entenda que seu objetivo foi alcançado]. Quando o usuário configura o idioma da plataforma e ao confirmar a operação, o designer informa através de uma frase que a ação foi realizada com sucesso. A frase, na cor verde, aparece dinamicamente na interface para informar que o usuário realizou essa função com sucesso.

[Você é um usuário que está habituado a acompanhar a execução do seu curso através de um calendário, para que à medida que for realizando as tarefas e assistindo os vídeos possa acompanhar o seu progresso. Como também o tempo restante para que você consiga concluir todo o módulo disponível]. O designer disponibiliza ao usuário uma linha do tempo. À medida que o usuário completa as etapas do seu curso MOOC, a linha inicialmente na cor cinza passa a ser preenchida pela cor verde. $\mathrm{O}$ que norteia o usuário, para que ele possa se organizar no planejamento de realização das tarefas do curso. Outro ponto a ressaltar é que o designer informa sempre ao usuário quanto tempo falta para que ele consiga concluir o módulo referente a semana e para informar ao usuário sobre o tempo que o resta o designer utiliza de um círculo cinza que começa a ser preenchido de verde, utilizando o mesmo padrão da linha do tempo (ver Figura 5).
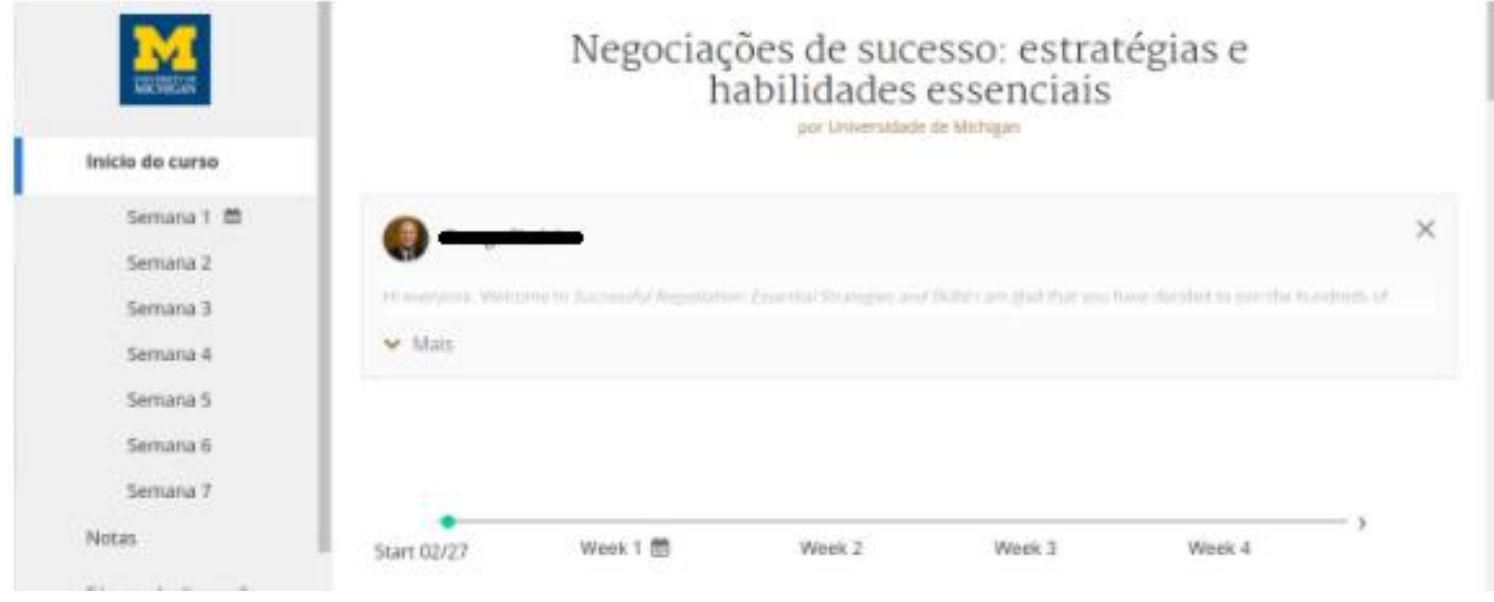

Figura 5: Linha do tempo.

\subsection{Resultados do Percurso Cognitivo}

Os passos necessários para a Tarefa 1 estão descritos a seguir.

\section{Tarefa 01: Cadastrar Usuário}

\section{Clicar no botão criar conta no canto superior direito.}

2. Inserir nome completo no primeiro campo texto. 
3. Inserir e-mail no segundo campo texto.

4. Inserir senha no terceiro campo texto.

5. Clicar no botão "Criar conta".

Na Tabela 1 encontram-se os resultados do PC referentes à Tarefa 1 . Na primeira coluna está situada a numeração de identificação referente à ordem das perguntas definidas pelo método PC. Os passos estão identificados com a letra "P" e o número identificador de sua sequência. As respostas estão assinaladas com as letras " $\mathrm{S}$ " para sim, " $\mathrm{T}$ " para talvez e "N" para não. Na Tarefa 1 os avaliadores não encontraram nenhum problema ao inspecionar a interface.

Tabela 1: Perguntas e respostas da tarefa cadastrar usuário.

\begin{tabular}{c|cc|c|c|c}
\multirow{2}{*}{$\begin{array}{c}\text { Perguntas / } \\
\text { Passos }\end{array}$} & \multicolumn{5}{|c}{ T1 } \\
\cline { 2 - 6 } & P1 & P2 & P3 & P4 & P5 \\
\hline 1 & $\mathrm{~S}$ & $\mathrm{~S}$ & $\mathrm{~S}$ & $\mathrm{~S}$ & $\mathrm{~S}$ \\
2 & $\mathrm{~S}$ & $\mathrm{~S}$ & $\mathrm{~S}$ & $\mathrm{~S}$ & $\mathrm{~S}$ \\
3 & $\mathrm{~S}$ & $\mathrm{~S}$ & $\mathrm{~S}$ & $\mathrm{~S}$ & $\mathrm{~S}$ \\
4 & $\mathrm{~S}$ & $\mathrm{~S}$ & $\mathrm{~S}$ & $\mathrm{~S}$ & $\mathrm{~S}$
\end{tabular}

Os passos necessários para a realização da segunda tarefa encontram-se descritos a seguir.

\section{Tarefa 02: Seleção de curso}

1. Clicar na barra que está escrito pesquisar lista de cursos.

2. Digitar a sigla MOOC.

3. Clicar no botão com símbolo de lupa.

4. Clicar no nome do curso (Negociações de sucesso: estratégias e habilidades essenciais).

5. Clicar no botão inscreva-se que está situado no lado esquerdo da tela.

6. Selecionar a opção "curso completo sem certificado".

7. Clicar em continuar.

8. Clicar no botão "Comece a aprender agora".

Na Tabela 2 pode ser visto que no P5 a pergunta de número 4 (se a ação for executada corretamente, o usuário vai perceber que está progredindo na direção de concluir a tarefa?) não foi respondida de forma afirmativa.

No P5, ao clicar no botão de inscrição no curso, o usuário esperará ser direcionado para a página com o conteúdo do curso, mas ao invés do conteúdo surgirá na tela uma caixa de texto com duas opções. As opções dividem-se em realizar o curso e pagar para receber o certificado ou realizar o curso gratuito sem o certificado. Esta pequena pausa pode surpreender os usuários por não conhecerem o modelo de negócio de um curso MOOC (Almeida et al., 2013), mas não deve impedir a interação dos estudantes. 
Tabela 2: Perguntas e respostas da tarefa seleção de curso.

\begin{tabular}{c|c|c|c|cc|c|c|c}
\multirow{2}{*}{$\begin{array}{c}\text { Perguntas } \\
\text { / Passos }\end{array}$} & & & \multicolumn{5}{|c|}{ T2 } \\
\cline { 2 - 9 } & P1 & P2 & P3 & P4 & P5 & P6 & P7 & P8 \\
\hline 1 & $\mathrm{~S}$ & $\mathrm{~S}$ & $\mathrm{~S}$ & $\mathrm{~S}$ & $\mathrm{~S}$ & $\mathrm{~S}$ & $\mathrm{~S}$ & $\mathrm{~S}$ \\
2 & $\mathrm{~S}$ & $\mathrm{~S}$ & $\mathrm{~S}$ & $\mathrm{~S}$ & $\mathrm{~S}$ & $\mathrm{~S}$ & $\mathrm{~S}$ & $\mathrm{~S}$ \\
3 & $\mathrm{~S}$ & $\mathrm{~S}$ & $\mathrm{~S}$ & $\mathrm{~S}$ & $\mathrm{~S}$ & $\mathrm{~S}$ & $\mathrm{~S}$ & $\mathrm{~S}$ \\
4 & $\mathrm{~S}$ & $\mathrm{~S}$ & $\mathrm{~S}$ & $\mathrm{~S}$ & $\mathrm{~N}$ & $\mathrm{~S}$ & $\mathrm{~S}$ & $\mathrm{~S}$
\end{tabular}

Para a Tarefa 3, os passos necessários estão descritos a seguir.

\section{Tarefa 03: Início do curso}

1. Descer a barra de rolagem.

2. Clicar no botão iniciar.

3. Passar o mouse nos botões situados no vídeo.

4. Clicar no botão com símbolo referente a legenda.

5. Selecionar a opção português (Brasil).

Na Tabela 3 estão as respectivas respostas às perguntas definidas para realização do PC. Nesta tabela, "T" significa "talvez", ocorrendo na realização do P3, quando a pergunta três é questionada ( $\mathrm{O}$ usuário vai associar a ação correta com o efeito que está tentando atingir?). Ao tentar sincronizar a legenda do vídeo, que não se encontra automaticamente sincronizada, o usuário deve buscar reportar o erro de sincronização da legenda. Reportar esse erro não é trivial, uma vez que a plataforma está configurada para o idioma português, mas partes da interface ainda continuam em inglês. Os avaliadores indicaram o talvez porque os botões do reprodutor de vídeo possuem tooltip ${ }^{6}$. Então, quando o usuário mover o cursor do mouse sobre os ícones, aparecerá uma ajuda contextual para informar a função de cada símbolo do reprodutor do vídeo. Possivelmente o usuário conseguirá entender essa ação e realizá-la sem dificuldades.

Já no $\mathrm{P} 4$, a resposta à pergunta “O usuário vai notar que a ação correta está disponível?" é negativa, pois o símbolo referente à lista de legendas não é semelhante a nenhum outro tipo comumente encontrado pelos avaliadores em outros reprodutores de vídeo online ou locais, como por exemplo, o Youtube ${ }^{7}$. Diferente do símbolo da lupa que é utilizado em outros sistemas para identificar a busca.

Tabela 3: Perguntas e respostas da tarefa início do curso.

\begin{tabular}{c|cc|c|c|c}
\multirow{2}{*}{$\begin{array}{c}\text { Perguntas } / \\
\text { Passos }\end{array}$} & \multicolumn{5}{|c}{ T3 } \\
\cline { 2 - 6 } & P1 & P2 & P3 & P4 & P5 \\
\hline 1 & $\mathrm{~S}$ & $\mathrm{~S}$ & $\mathrm{~S}$ & $\mathrm{~S}$ & $\mathrm{~S}$ \\
2 & $\mathrm{~S}$ & $\mathrm{~S}$ & $\mathrm{~S}$ & $\mathrm{~N}$ & $\mathrm{~S}$ \\
3 & $\mathrm{~S}$ & $\mathrm{~S}$ & $\mathrm{~T}$ & $\mathrm{~S}$ & $\mathrm{~S}$ \\
4 & $\mathrm{~S}$ & $\mathrm{~S}$ & $\mathrm{~S}$ & $\mathrm{~S}$ & $\mathrm{~S}$
\end{tabular}

\footnotetext{
${ }^{6}$ Tooltip ou dica de contexto é um elemento comum de interface gráfica. Tooltip é uma moldura flutuante que surge quando o cursor do mouse está sobre um elemento da interface e contém uma explicação adicional sobre tal elemento. 7 https://www.youtube.com/
} 
Por fim, os passos necessários à realização da última tarefa encontram-se a seguir.

\section{Tarefa 04: Participação no fórum}

1. Clicar na frase "Alguma pergunta? Discutir essa videoaula nos fóruns da semana".

2. Clicar no botão com símbolo de novo tópico.

3. Inserir um nome descritivo para um novo tópico.

4. Fornecer detalhes ou o contexto do novo tópico.

5. Selecionar o fórum (qual etapa deseja discutir).

6. Clicar no botão publicar.

Na Tabela 4 encontram-se as respostas referente à Tarefa 04 . O P5 foi o único passo em que os avaliadores encontraram problemas, especialmente nas perguntas 01 ("O usuário vai tentar atingir o efeito correto?") e 03 ("O usuário vai associar a ação correta com o efeito que está tentando atingir?"). Ao selecionar a opção "Novo fórum" e inserir as informações padrão, o estudante depara-se com uma lista que "solicita" a seleção de um fórum. Essa opção não indica que tipo de resposta é necessária para a seleção do fórum.

A resposta à pergunta 03 foi definida como talvez, pois o usuário só identifica o que precisar ser selecionado quando a lista do fórum aparecer, após o clique do usuário. A lista de opções não está clara para o usuário.

Tabela 4: Perguntas e respostas da tarefa participação no fórum.

\begin{tabular}{c|c|c|c|cc|c}
\multirow{2}{*}{$\begin{array}{c}\text { Perguntas } \\
\text { / Passos }\end{array}$} & & & & T4 & \\
\hline & P1 & P2 & P3 & P4 & P5 & P6 \\
\hline 1 & $\mathrm{~S}$ & $\mathrm{~S}$ & $\mathrm{~S}$ & $\mathrm{~S}$ & $\mathrm{~N}$ & $\mathrm{~S}$ \\
2 & $\mathrm{~S}$ & $\mathrm{~S}$ & $\mathrm{~S}$ & $\mathrm{~S}$ & $\mathrm{~S}$ & $\mathrm{~S}$ \\
3 & $\mathrm{~S}$ & $\mathrm{~S}$ & $\mathrm{~S}$ & $\mathrm{~S}$ & $\mathrm{~T}$ & $\mathrm{~S}$ \\
4 & $\mathrm{~S}$ & $\mathrm{~S}$ & $\mathrm{~S}$ & $\mathrm{~S}$ & $\mathrm{~S}$ & $\mathrm{~S}$
\end{tabular}

As seguintes melhorias foram propostas a partir dos problemas encontrados com o PC:

- padronizar o idioma da plataforma;

- sincronizar a legenda automaticamente de acordo com o idioma selecionado para o site;

- diminuir os passos para que o usuário consiga inscrever-se no curso;

- modificar o nome fórum na criação de um novo tópico para algo mais intuitivo.

\section{Discussão dos Resultados}

Nesta seção são apresentados os resultados da terceira etapa do estudo, que é a correlação dos resultados com os dados apresentados na execução do MIS e do PC com as causas de abandono de estudantes da plataforma Coursera.

Os avaliadores não encontraram dificuldades para a realização dos dois métodos propostos, utilizados com propósito de avaliar a plataforma de um curso MOOC sob o enfoque da usabilidade e comunicabilidade integralizadas. Os dois métodos de avaliação explicitaram a deficiência da plataforma no tocante à seleção do idioma, por exemplo. Mesmo o idioma português tendo sido selecionado, algumas partes do curso e da plataforma não foram traduzidas, 
relacionando-se a última causa citada de abandono, o idioma da plataforma. Como pode ser visto na Figura 5, em que a linha do tempo está cada semana indicada como Week.

Esse aspecto é considerado problema de comunicabilidade, pois a falha da tradução não está relacionada ao conteúdo do curso, que poderá estar disponibilizado apenas no idioma de concepção do curso, mas à deficiência no suporte da plataforma às suas próprias configurações de trocas de idioma.

Outra ruptura encontrada por meio do PC, foi na tarefa de criação de novo tópico do fórum. Não existe uma opção que evidencie ao usuário qual seria o próximo passo necessário para a realização da tarefa. Isso pode estimular a desistência da criação do tópico ou a criação do tópico em uma sessão errada do curso. Consequentemente, ocasionará a falta de retorno ou interação do(s) estudante(s) nos fóruns, seja por não ter feedback do tutor/professor ou dos pares. Esta falha relaciona-se ao motivo de abandono relacionado à privação de interação entre aluno e professor. A ausência de feedback faz com que a experiência de um curso massivo se torne paradoxal, pois mesmo os MOOC possuindo milhares de participantes, a interação individual é solitária, por ser autodirigida (Carmo, 2017).

Ressalta-se que em um curso MOOC o estudante é informado se o curso utiliza vídeos previamente gravados, não sendo exibidas aulas ao vivo do professor; portanto, sem interação direta. A interação ocorre assincronamente por meio de fóruns com outros estudantes, uma característica incentivada nos MOOC.

Na realização do MIS, notou-se que a legenda do vídeo não estava sincronizada. Para que o erro fosse corrigido, foi necessário buscar ajuda no símbolo estático que referenciava a imagem de uma bandeira, associado pelos avaliadores como um sinal de socorro. Esse problema de sincronização não ocorreu com a realização do PC, mas o símbolo referente à legenda parece não ser intuitivo para o usuário, podendo ser identificado como listagem de idiomas. Caso não ocorra adequação no uso de tecnologias, o estudante talvez não consiga sincronizar a legenda ou configurar o idioma, podendo ocasionar o abandono.

Podemos supor que, caso um estudante possua limitações no uso de Tecnologia da Informação (TI), pode vir a abandonar o curso. Porque mesmo os MOOC sendo denominados "abertos", os estudantes devem possuir duas competências básicas para a sua utilização, sendo uma delas habilidades no uso de tecnologias da informação (Fini, 2009).

Ao realizar o MIS, foi identificada a existência de um símbolo informando por quanto tempo os módulos do curso ficarão disponíveis. Ao lado dessa informação há um símbolo, representado por um livro aberto, com a frase "nada expirado". A interpretação do símbolo remete à limitação temporal do material do curso. Isso está relacionado à escassez de tempo, uma das causas de abandono de cursos MOOC. Nessa situação, o usuário precisa se esforçar para conseguir finalizar o módulo antes que o tempo de acesso ao material expire.

O principal motivo para o abandono de estudantes em MOOC identificado no estudo de (Nawrot \& Doucet, 2014) foi o mau gerenciamento do tempo, dado esse confirmado por $68,9 \%$ dos participantes que responderam à pesquisa. Outros artigos reafirmam que a falta de tempo por parte dos estudantes é um fator determinante no abandono dos cursos MOOC (Burd et al., 2015; Fini, 2009; Zheng et al., 2015).

A causa referente à dificuldade em expor ideias de maneira clara numa comunicação escrita não foi correlacionada com nenhum dos problemas encontrados. Ao término dessa etapa da pesquisa, notou-se que a plataforma Coursera disponibiliza diversos atalhos para a central de ajuda; contudo, inglês é a língua predominante. Caso o usuário não tenha domínio no idioma terá dificuldades. 
De modo geral, ambos os métodos foram importantes para a avaliação da plataforma MOOC, pois os problemas encontrados na execução do MIS não foram identificados na execução do PC, e vice-versa. Ambos os métodos possuem objetivos diferentes, mas necessários para uma boa interação do estudante com o curso MOOC.

\section{Considerações Finais}

Neste artigo relatamos uma avaliação híbrida da comunicabilidade e usabilidade da plataforma Coursera, utilizando o MIS e o PC, com o objetivo de investigar problemas que poderiam estar relacionados com causas de abandono dos estudantes em MOOC. Algumas rupturas de comunicabilidade encontradas na execução do MIS foram correlacionadas com motivos que ocasionam abandono dos cursos e confirmadas com os resultados do PC.

As inspeções por intermédio dos métodos supracitados indicaram diversas modificações que podem ser realizadas na plataforma Coursera para favorecer a usabilidade e comunicabilidade do sistema. É possível que a adoção dessas melhorias propicie aumento do número de concluintes nos cursos disponíveis na plataforma. Principalmente pelo caráter holístico do uso de dois critérios de qualidade em IHC na avaliação do sistema.

Com a avaliação de comunicabilidade e usabilidade identificou-se rupturas/falhas que podem vir a prejudicar a interação dos estudantes. Embora a maioria das causas tenham sido confirmadas com os resultados dos métodos, descobriram-se apenas indícios; sendo, portanto, necessárias novas avaliações que envolvam diretamente os usuários/estudantes de MOOC, a partir de experimentos ou estudos de caso.

Espera-se que os resultados das inspeções sejam convertidos em melhorias necessárias em futuras versões da interface do Coursera, corrigindo as falhas encontradas. Para que a interface se torne adequada à experiência do estudante (usuário) em seu processo de aprendizagem com uma interação agradável e inteligível. Nesse sentido, pretendemos contatar o Coursera para apresentar as sugestões de melhorias com base nas avaliações.

Os tópicos apresentados como falhas de usabilidade ou comunicabilidade podem ser utilizados como requisitos de usuário para o desenvolvimento de novas plataformas de MOOC. Ademais, é possível replicar a abordagem conduzida neste trabalho em outras plataformas, como Udemy, edX, para ampliar e validar os requisitos elencados até o momento. A disponibilização de diretrizes para o desenvolvimento de interfaces de plataformas MOOC adequadas às necessidades dos usuários por meio dos resultados da inspeção pode ser uma importante contribuição para comunidade, especialmente de Computação e Educação.

Em trabalhos futuros, pretende-se aplicar outros métodos de avaliação da interação, como o Método de Avaliação de Comunicabilidade (MAC), com o objetivo de avaliar rupturas de comunicabilidade com o auxílio do usuário; também desenvolver um estudo de caso de implantação de um MOOC avaliando o processo de criação em tempo de design e em tempo de interação, visando aumentar a qualidade da metacomunicação e consequentemente investigar se haverá um aumento na taxa de permanência dos estudantes de MOOC. Por fim, esperamos adicionar na abordagem avaliativa o uso de uma checklist fundamentada na WCAG (Web Content Accessibility Guidelines) para verificar a adequação das plataformas de MOOC quanto à adequação da acessibilidade nas interfaces. 


\section{Agradecimentos}

Agradecemos à Coordenação de Aperfeiçoamento de Pessoal de Nível Superior (CAPES) por meio do programa CAPES-DS (\#88882.453912/2019-01, \#88882.453913/2019-01 e \#88887.464930/2019-00) e ao Grupo de Pesquisa e Extensão em Informática, Educação e Sociedade - Onda Digital - da Universidade Federal da Bahia.

\section{Referências}

Alario-Hoyos, C., Pérez-Sanagustín, M., Delgado-Kloos, C., Parada, H. A., Muñoz-Organero, G. M., \& Rodríguez-de-las-Heras, A. (2013). Analysing the impact of built-in and external social tools in a mooc on educational technologies. In European Conference on Technology Enhanced Learning - Springer. p. 5-18. doi: 10.1007/978-3-642-40814-4_2 [GS Search]

Almeida, O. C. d. S., Abbad, G., Meneses, P. P. M., \& Zerbini, T. (2013). Evasão em cursos a distância: fatores influenciadores. Revista Brasileira de Orientação Profissional, 14(1), p. 19-33. [GS Search]

Armstrong, L. (2013). The year of ups and downs for the MOOCs. Changing Higher Education. Disponível em: http://goo.gl/SqwGWn. Acesso em: 25/11/2020.

Barbosa, S. D. J., Silva, B. S. d. (2010). Interação humano-computador. Elsevier Brasil. [GS Search]

Baloi, J. A. (2009). A concepção da educação democrática na obra "Democracia e Educação" de Jonh Dewey. Monografia-Faculdade de Filosofia e Ciências Humanas da Universidade São Tomás de Moçambique, Maputo.

Bartolomé, A. (2015). Are MOOCs Promising Learning Environments?/¿ Son los MOOC una alternativa de aprendizaje?. Comunicar (English edition), 22(44), p. 91-99. doi: 10.3916/C442015-10 [GS Search]

Baturay, M. H. (2015). An overview of the world of MOOCs. Procedia-Social and Behavioral Sciences, 174, p. 427-433. doi: 10.1016/j.sbspro.2015.01.685 [GS Search]

Belanger, Y., Thornton, J., \& Barr, R. C. Bioelectricity. (2013). A quantitative approach--Duke University's first MOOC. EducationXPress, 2013(2), p. 1-21. [GS Search]

Bittencourt, G. P. (2011). Evasão na educação a distância do ensino superior: estudo de caso no $1^{\circ}$ curso de administração EAD da UFRGS. Trabalho de Conclusão de Curso. Universidade Federal do Rio Grande do Sul. [GS Search]

Burd, E. L., Smith, S. P., \& Reisman, S. (2015). Exploring business models for MOOCs in higher education. Innovative Higher Education, 40(1), p. 37-49. doi: 10.1007/s10755-014-9297-0 [GS Search]

Burge, J. (2015). Insights into teaching and learning: Reflections on mooc experiences. Proceedings of the 46th ACM Technical Symposium on Computer Science Education. p. 600603. doi: 10.1145/2676723.2677243 [GS Search]

Carmo, T. M. (2017). Como se aprende num MOOC? Revista da UIIPS, Instituto Pilitécnico de Santarem, 5(2), p. 198-210. doi: 10400.15/2330 [GS Search]

Clow, D. (2013). Moocs and the funnel of participation. In Proceedings of the Third International Conference on Learning Analytics and Knowledge, ACM. p 185-189. doi: $\underline{10.1145 / 2460296.2460332}$ [GS Search] 
De Souza, C. S., \& Leitão, C. F. (2009). Semiotic engineering methods for scientific research in HCI. Synthesis Lectures on Human-Centered Informatics, 2(1), p. 1-122. doi: 10.2200/S00173ED1V01Y200901HCI002 [GS Search]

Do Rêgo, B. B., Garrido, F., \& Matos, E. (2017a). Identifying influences of the quality of interaction on dropout rates of MOOC: preliminary results. In Proceedings of the XXVIII Brazilian Symposium on Computers in Education (Simpósio Brasileiro de Informática na Educação-SBIE), p. 1766-1768. doi: 10.5753/cbie.sbie.2017.1766 [GS Search]

Do Rêgo, B. B; Monteiro, I. T; Sampaio, A. L. (2017b). Communicability evaluation of privacy settings on Facebook for Android. In International Conference on Human Aspects of Information Security, Privacy, and Trust. Springer, Cham. p. 623-639. doi: 10.1007/978-3319-58460-7 43 [GS Search]

Do Rêgo, B. B., Garrido, F., Rosa, J., \& Matos, E. (2019). Communicability problems on MOOCs: a study around metacommunication. In Proceedings of the XXX Brazilian Symposium on Computers in Education (Simpósio Brasileiro de Informática na Educação-SBIE), p. 12611270. doi: $10.5753 /$ cbie.sbie.2019.1261 [GS Search]

Downes, S. (2012). Connectivism and connective knowledge: Essays on meaning and learning networks. Stephen Downes Web.

Dinniz, D. D. (2007). A Interação no Ensino à Distância sob a Ótica dos Estilos de Aprendizagem. Tese de Doutorado. Universidade de São Paulo. [GS Search]

Fini, A. (2009). The technological dimension of a massive open online course: The case of the CCK08 course tools. International Review of Research in Open and Distributed Learning, 10(5), p. 1- 27. doi: 10.19173/irrodl.v10i5.643 [GS Search]

Gomez-Zermeno, M. G., \& De La Garza, L. A. (2016). Research analysis on mooc course dropout and retention rates. Turkish Online Journal of Distance Education, pages 3-14. doi: 10.17718/tojde.23429 [GS Search]

Grainger, B. (2013). Introduction to MOOCs: avalanche, illusion or augmentation. Policy Brief UNESCO (july). Institute for Information Technologies in Education. (URL: http://iite.unesco.org/pics/publications/en/files/3214722.pdf). [GS Search]

He, J., Bailey, J., Rubinstein, B. I., \& Zhang, R. (2015). Identifying at-risk students in massive open online courses. In AAAI Conference on Artificial Intelligence, p. 1749-1755. doi: $\underline{10.5555 / 2886521.2886563}$ [GS Search]

Nawrot, I., \& Doucet, A. (2014). Building engagement for MOOC students: introducing support for time management on online learning platforms. In: Proceedings of the 23rd International Conference on world wide web. p. 1077-1082. doi: 10.1145/2567948.2580054 [GS Search]

Onah, D. F. O., Sinclair, J., \& Boyatt, R. (2014). Dropout rates of massive open online courses: behavioural patterns. In Proceedings EDULEARN14, p. 5825-5834. doi: 10.13140/RG.2.1.2402.0009 [GS Search]

Pappano, L. (2012). The Year of the MOOC. The New York Times, v. 2, n. 12, p. 2012. [GS Search]

Salgado, L. C. d. C., \& De Souza, C. S. (2007). CommEST-Uma ferramenta de apoio ao método de Avaliação de Comunicabilidade. In: III Conferência Latino-Americana de Interação Humano-Computador. [GS Search]

Salgado, L. C. d. C., Bim, S. A., \& De Souza, C. S. (2006). Comparação entre os métodos de avaliação de base cognitiva e semiótica. In: Proceedings of VII Brazilian symposium on 
Human factors in computing systems. ACM. p. 158-167. doi: $\underline{10.1145 / 1298023.1298045}$ [GS Search]

Silveira, M. S., de Souza, C. S., \& Barbosa, S. D. (2003). Um método da engenharia semiótica para a construção de sistemas de ajuda online. In Latin American Conference On Humancomputer Interaction. Rio de Janeiro. V. 17, p. 167-177. [GS Search]

Zheng, S., Rosson, M. B., Shih, P. C., \& Carroll, J. M. (2015). Understanding student motivation, behaviors and perceptions in MOOCs. In Proceedings of the 18th ACM conference on computer supported cooperative work \& social computing. $p . \quad \mathrm{S} / \mathrm{N}$. doi: $\underline{10.1145 / 2675133.2675217}$ [GS Search] 